\title{
Experimental investigation of a round jet impacting a disk engraved with radial grooves
}

\author{
Sofiene Ouled Taleb Salah ${ }^{\mathrm{a}, \mathrm{b}, *}$, Alexis Duchesne ${ }^{\mathrm{a}}$, Nicolas De Cock ${ }^{\mathrm{b}}$, Mathieu Massinon ${ }^{\mathrm{b}}$, \\ Khaled Sassi ${ }^{\mathrm{c}}$, Khaoula Abrougui ${ }^{\mathrm{d}}$, Frédéric Lebeau ${ }^{\mathrm{b}}$, Stéphane Dorbolo ${ }^{\mathrm{a}}$ \\ a University of Liege, CESAM - GRASP, Institute of Physics, Building B5a, Sart Tilman, 4000 Liege, Belgium \\ ${ }^{\mathrm{b}}$ University of Liege, TERRA Teaching and Research Center, Gembloux Agro-Bio Tech, Passage des déportés 2, 5030 Gembloux, Belgium \\ ${ }^{c}$ University of Carthage, Laboratory of Agronomy, National Agronomic Institute of Tunisia (INAT), 1082 Tunis, Tunisia \\ ${ }^{\mathrm{d}}$ Department of Horticultural Systems Engineering, Higher Institute of Agronomy, 4042 Sousse, Tunisia
}

\section{A R T I C L E I N F O}

\section{Article history:}

Received 6 July 2017

Received in revised form 12 June 2018

Accepted 19 June 2018

Available online 26 June 2018

\section{Keywords:}

Groove

Phase diagram

Turbulent liquid sheets

Turbulent atomization of liquid sheets

Jet numbers

Droplet diameters

Droplet velocities

\begin{abstract}
A B S T R A C T
The present work proposes to investigate the impact of a turbulent round jet on a disk. The disk diameter is one order of magnitude larger than the jet diameter but small enough to avoid the formation of a circular hydraulic jump. The case of a smooth disk is first studied as the reference case. We then report results obtained with a disk engraved along its circumference by a number $N$ of radial grooves. The grooves are used to split the liquid sheet into multiple jets. According to the incoming flow rate $Q$ and to the geometry of the groove, the number of jets $n$ can be stable and corresponds to $2 N$ jets and $N$ jets, or variable, i.e. merged jets (mixed zone). Phase diagrams $(Q, n)$ are deduced from measurements for different lengths of the groove. Finally, the obtained droplets are characterized in terms of diameters and velocities.
\end{abstract}

(c) 2018 Elsevier Masson SAS. All rights reserved.

\section{Introduction}

Spray application techniques in agriculture use droplet clouds to carry the active ingredient to the target area [1]. Pesticide application efficiency as a function of droplet sizes and velocities has been the subject of an extensive investigation and despite the process complexity some trends are well established [1,2]. As a rule of thumb, droplets whose diameters exceed $300 \mu \mathrm{m}$ have the tendency to splash on the target surface. Among others, the parameters determining the splash characteristics are the droplet kinetic energy and the surface properties [3-5]. On the other hand, small droplets under $200 \mu \mathrm{m}$ are prone to interactions with the wind which may cause their drift away from the target $[1,2]$. However, agricultural nozzles are characterized by a wide distribution of droplet size (span) involving potential drifting or efficiency losses due to splashing phenomena. Thus, the spray should contain optimal droplets with regard to their sizes and velocities. A better spray uniformity may improve treatment efficiency and reduce drift hazard. On this basis, reducing the extent of the droplet size distribution is still a challenge in the field of agricultural hydraulic nozzles.

\footnotetext{
* Corresponding author at: University of Liege, CESAM-GRASP, Institute of Physics, Building B5a, Sart Tilman, 4000 Liege, Belgium.

E-mail address: s.ouled@ulg.ac.be (S. Ouled Taleb Salah).
}

As the simplicity, the robustness, the low cost are required, a round jet impacting vertically a horizontal smooth motionless disk is the ideal candidate for the massive production of droplets. The pioneer study on the subject was conducted by Savart, who observed many phenomena depending, among other parameters, on the ratio $(X)$ : the impacted disk diameter $D$ divided by the jet diameter $d\left(X=\frac{D}{d}\right)[6-11]$.

In the case $\boldsymbol{X}=\mathbf{0}$, a circular orifice generates a cylindrical jet with a diameter $d$ and an initial velocity $U_{0}$ where $U_{0}=\frac{4 Q}{\pi d^{2}}$ (with $Q$ the injected flow rate). This jet is characterized by two dimensionless numbers: the Reynolds number $\operatorname{Re}=\frac{U_{0} d}{v}$ (with $v$ the kinematic viscosity) and the Weber number $W e=\frac{\rho d U_{0}^{2}}{\sigma}$ (with $\rho$ the liquid density and $\sigma$ the liquid surface tension). The jet fragmentation occurs due to the capillary instability depending on $U_{0}$. It follows the Plateau-Rayleigh break up mode for which the most unstable perturbation breaks the jet in droplets of 1.89 times the jet diameter [12-14].

When $\boldsymbol{X} \sim \mathbf{1}$, the jet impacts the plate center with a velocity $U_{0}$ and is forced to spread out radially, creating a liquid film. At the disk edge, the inertia force tends to detach the film whereas wetting forces try to keep the film on the disk. Once the liquid film detaches at the disk edge, it forms an ejection angle with the disk. This ejection angle is sensitive to $X$ and $U_{0}$ [15]. One cannot reach an ejection angle of $\frac{\pi}{2}$ with a flat impactor [15]. Finally, the 
radius of the liquid sheet $R$ is determined by a balance between the flow inertia and capillary forces $[11,16]$. Within a laminar regime, the maximal radial extension $R$ is given by the non dimensional diameter $\frac{2 R}{d}=\frac{W e}{8} \frac{U}{U_{0}}\left(1-\frac{V_{d}}{U}\right)(U$ is the film velocity at the disk edge and $V_{d}$ is the emitted droplet velocity) [9]. In the limit $\frac{V_{d}}{U} \ll 1$ and $\frac{U}{U_{0}}=1$, one finds $\frac{2 R}{d}=\frac{W e}{8}$ (i.e the case of a constant thickness sheet with no initial velocity) [16]. On the other hand, data relative to the turbulent regime are scarce. A competition between the velocity of the radial flow and the acceleration due to the gravity is also involved in this phenomenon and is governed by the dimensionless Froude number $F r=\frac{U}{\sqrt{g h}}$ (with $U$ the mean flow velocity, $g$ the gravity acceleration and $h$ the liquid thickness): If $\mathrm{Fr}$ is higher than 1 , the effect of the gravity acceleration on the liquid particle trajectory can be neglected and the liquid sheet remains flat.

Depending on the $W e$, two regimes can be distinguished. Below a critical value $W e_{c}(\approx 1000)$, the effect of the surrounding air on the dynamics of the sheet can be neglected. The liquid sheet keeps a smooth aspect and remains planar up to the edge and hence a smooth regime is observed [9]. When We is superior to $W e_{c}$, a flapping regime is obtained and the sheet edge is moving up and down like a flag flapping in the wind [10].

A lot of experimental, numerical and theoretical studies have been principally devoted to the liquid film prior to the droplet formation, describing the velocity and the thickness of this film [1719] but also the dynamics of the liquid sheet and its collapse [9,11]. The transition from sheet to drops has also been addressed: The drops are ejected at the extremities of the cusp indentations located on the sheet rim through a Savart-Plateau-Rayleigh instability. The drops are attached at the liquid sheet by capillary forces and they are also subject to the centrifugal acceleration. Then, the drops are detached when the acceleration effect overcomes the capillary force; they are thus released with various sizes and velocities $[9,11]$. The droplet diameter decreases with the We, first slowly for $W e$ inferior to $W e_{c}=1200$ and more strongly for higher We values [10].

For $\boldsymbol{X} \rightarrow \infty$, a circular hydraulic jump is observed [17,20,21]. The hydraulic jump is the sudden transition from a supercritical liquid flow (high velocity and low liquid thickness) to a subcritical flow (low velocity and high thickness). This transition is characterized by an almost vertical liquid wall named hydraulic jump. The hydraulic jump depends critically on the Fr value [21].

As explained before, the droplet size is a key parameter for agriculture issue and other configurations has been proposed. For example, rotating cups where centrifugal forces allow the generation of cylindrical jets having almost homogeneous diameters and velocities. The jets break up according to the Rayleigh-Plateau and droplet sizes are simply tuned by altering the rotation speed and the fed flow rate. These droplets are emitted with velocities between $15 \mathrm{~m} \mathrm{~s}^{-1}$ and $25 \mathrm{~m} \mathrm{~s}^{-1}$. This is the basis of the Controlled Droplet Application (CDA) concept in agriculture that has been developed using rotary atomizers [1]. The bulkiness of these devices has dramatically reduced their use except in some specific applications.

We propose to study the influence of deep radial grooves (order of magnitude of the liquid film thickness) with controlled shapes. Some textured surfaces were already used to study their effects on the dynamics of liquid sheet. The presence of micrometer-sized posts regularly arranged on the impactor influenced the water bell shape as it adopted polygonal shapes on its rim [22]. In addition, Taylor investigated the effect of grooves engraved radially and regularly on the disk surface [23]. The grooves were knife cuts. As a result, some structures appeared under the form of cardioids on the liquid sheet. However, such a groove geometry was not sufficient to destabilize the sheet. In this paper, we will show that, under given conditions of the flow, the sheet destabilizes into jets. The geometrical parameters of the disk will be tuned as its diameter, the size of the grooves (depth, length, width) and their numbers. To obtain enough jets one needs an impact disk of intermediary size (typically $X \sim 10$ ): large enough to engraved a lot of grooves but small enough to avoid hydraulic jump and remain in the Savart sheet regime.

The paper is structured as follows: In the first part the situation of a liquid jet impacting a smooth centimetric disk $(10 \mathrm{~mm}, 20 \mathrm{~mm}$, $25 \mathrm{~mm}, 30 \mathrm{~mm}$ and $40 \mathrm{~mm}$ ) is addressed. This constitutes our basis cases to which results on engraved disks are to be confronted. Then, in the second part, the $30 \mathrm{~mm}$ disk engraved along its circumference by radial grooves in order to induce the split of the film into multiples jets is studied. Several regimes are described and transitions are detailed through experimental phase diagrams. The generated droplets are then characterized in terms of sizes and velocities.

\section{Experimental setup and used techniques}

\subsection{Setup description}

The whole experimental setup is presented in Fig. 1. The nozzle geometry consisted in a glass injector (characterized by an internal diameter of $3 \mathrm{~mm}$ and a length of $155 \mathrm{~mm}$ ) set perpendicularly to a horizontal motionless disk. The orifice injector generated a cylindrical liquid jet $d=3 \mathrm{~mm}$ which impacted the disk center with a mean velocity $U_{0}$. The disk was constituted by a plexiglas plate cut with a laser cutter. The disk diameters $D$ were : $10 \mathrm{~mm}$, $20 \mathrm{~mm}, 25 \mathrm{~mm}, 30 \mathrm{~mm}$ and $40 \mathrm{~mm}$. The disk under consideration was fixed on a support in the middle of a $200 \mathrm{~mm}$ side cubic tank and its horizontality was checked by a spirit level. The gap $H$ between the injector tip and the disk surface was tuned by a three axis precision displacement tool (XYZ). In the following experiments, the gap was set to $550 \mu \mathrm{m}$ that represents the critical distance that can be achieved with regards to the gear pump.

The liquid flow was injected using a gear pump (Ismatec BVP-Z) combined with a pump head to reduce the flow pulsation and thus delivering steady liquid flows. The flow was measured by weighing the liquid poured into a graduated tank during a specific time (30 s) and three repetitions were performed for each flow measurement. By changing its rotation speeds, flow rates $Q$ were varied from 1.5 $10^{-5} \mathrm{~m}^{3} \mathrm{~s}^{-1}$ to $4.110^{-5} \mathrm{~m}^{3} \mathrm{~s}^{-1}$.

The used liquid was tap water at $20{ }^{\circ} \mathrm{C}$ (density $\rho=$ $1000 \mathrm{~kg} \mathrm{~m}^{-3}$, kinematic viscosity $v=10^{-6} \mathrm{~m}^{2} \mathrm{~s}^{-1}$ and surface tension $\sigma=73.5 \pm 0.3 \mathrm{mN} \mathrm{m}^{-1}$ ). The wetting property of the water on the plexiglass surface was characterized by a measured contact angle of $74^{\circ}$ (static wetting) measured using an optical contact angle meter (CAM $200 \mathrm{KSV}$ ).

In order to acquire images of the liquid film on the disk border and of emitted droplets, a high speed camera Y4 (IDT motion) was used to record the fluid motion from the top. A mirror was tilted by $45^{\circ}$ to optic axis and it reflected the light emitted from the source light which was set perpendicularly to the mirror (Fig. 1). The images were acquired at a frequency of $2000 \mathrm{~Hz}$ and treated with a Matlab code.

\subsection{Film thickness measurement}

To measure the thickness of the liquid film, we chose the technique described by Azuma and Toshino [18]. A thin needle driven by a three axis precision displacement tool (XYZ) was set $1 \mathrm{~mm}$ downstream before the disk edge. The needle was lowered down and when it touched the liquid surface, a droplet was rapidly formed. Then, the liquid flow was stopped and the needle was lowered down to reaching the disk surface (Fig. 3). The difference 


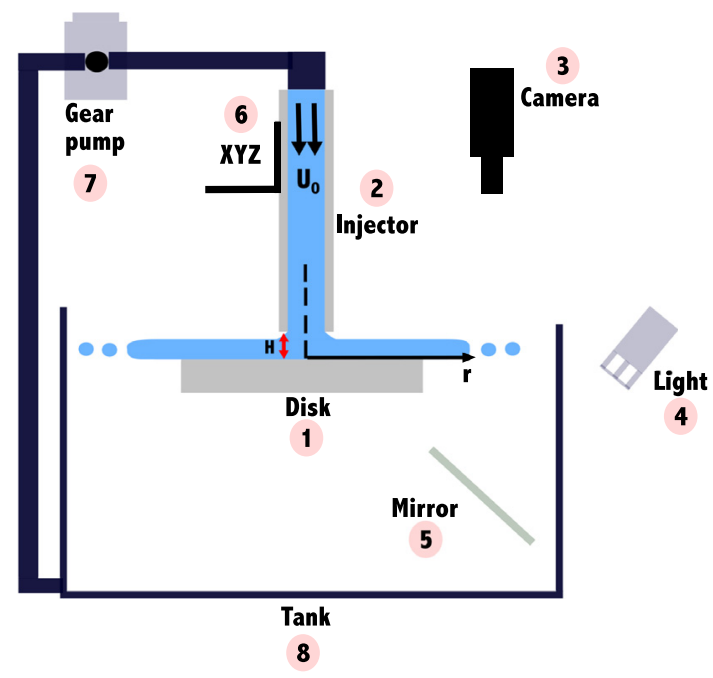

Fig. 1. Experimental setup: An injector consisting of a glass pipe (2) generated a round jet $d=3 \mathrm{~mm}$. The injected water impacted the center of a plexiglass disk (1) with a velocity $U_{0}$. The disk was placed in the middle of a $200 \mathrm{~mm}$ side cubic tank (8). The liquid then spread out in a radial distance along $r$ axis. The gap $H$ between the injector tip and the disk was fixed using a precision displacement tool XYZ (6). A high-speed camera (3) was mounted vertically to the disk border to acquire images of liquid sheet and emitted droplets. A mirror (5) was tilted by $45^{\circ}$ to the camera optic axis in order to reflect the light towards the disk. The liquid was injected by the gear pump (7) connecting the tank to the injector (2).

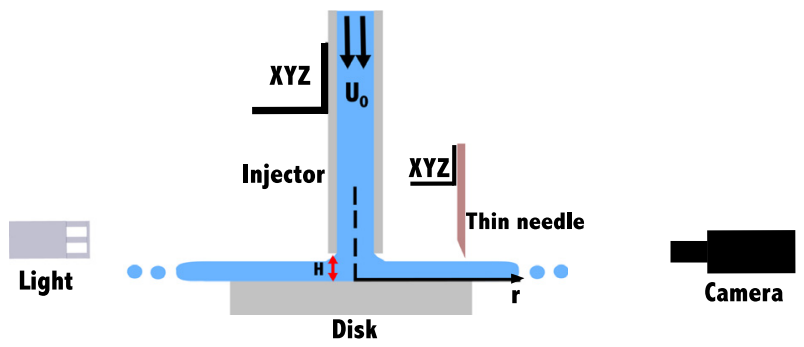

Fig. 2. Experimental setup for film thickness measurement: A thin needle set on the liquid flow downstream ( $1 \mathrm{~mm}$ before the disk border from the jet impact center) was used to measure the thickness of liquid film. Its displacement was tuned by a precision three axis displacement tool. A high-speed camera Y4, for which the optic axis was set perpendicularly to the thin needle axis and facing a light source, was used to control the measurement process.

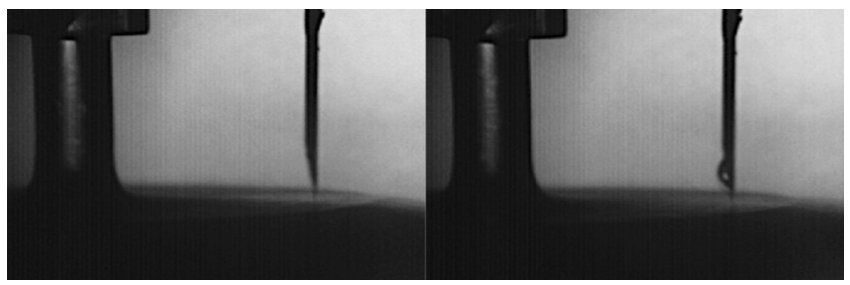

Fig. 3. Illustration of the film thickness measurement process based on a thin needle displacement. The needle was set on the flow downstream ( $1 \mathrm{~mm}$ before the disk edge). On the right, the needle touched the liquid surface and a small droplet appeared rapidly. The Re and We were respectively 10640 and 529.

between both heights gives the liquid thickness. The measurement process was eye-guided using the images from the high-speed camera Y4 optic axis which was set perpendicularly to the needle axis, on the same plane as the disk surface (Fig. 2). Using this film thickness measurement, the film mean velocity reads: $U(r)=\frac{Q}{2 \pi r h}$ (with $U$ : film mean velocity, $Q$ : injected flow rate, $r$ : flow radial distance and $h$ : film thickness).

\subsection{Droplet size measurement}

A high-speed camera Y4 coupled to a LED light recorded image shadows of water droplets. Droplet size measurements were carried out at $12 \mathrm{~mm}$ from the droplet ejection region $(80 \mathrm{~mm}$ from the edge of the smooth disk and $25 \mathrm{~mm}$ from the edge of the engraved disk). This distance was chosen in order to avoid the jet break up zone and to obtain less dense droplet clouds. It also allowed one to consider the final droplets distribution, after merging phenomena happened. The images acquisition frequency was $5000 \mathrm{~Hz}$. Images were analyzed with a Particle Tracking Velocimetry Sizing (PTVS) algorithm developed in Matlab and provided droplet size and velocity components perpendicular to optic axis [24]. The statistics of the droplet measurements were established for a number of drops superior to 10000 .

\section{Impact on a smooth disk}

\subsection{Qualitative view}

When the tap water jet $(d=3 \mathrm{~mm}$ ) impacted the disk center, the water spread out radially and formed a thin film on the disk surface. At the edge of the plate, the liquid film was ejected almost horizontally (not subject to the gravity) and formed a Savart sheet of radius $R$. This sheet was delimited by nodes where the liquid was concentrated. These nodes were the emission point of droplets (Fig. 4) as observed in the literature [11]. The Re numbers inside the injector has been calculated to range between 9000 and 16000 which correspond to a turbulent impact on the disk. Hence, the sheet and its rim were turbulent by construction. Impact wave disturbances emanating from the turbulent impact may be observed on the sheet surface. As a result, the local disturbances in the sheet triggered the formation of holes (or perforations) in the liquid sheet (outside of the disk), which amplified and lead to the disintegration of the liquid sheet (Fig. 4). Studies on the turbulent regime are relatively scarce. However, these holes were observed within the turbulent splash plate atomization [25].

Fig. 5 shows the evolution of $\frac{2 R}{d}$ of the water sheet as a function of the We for different disk diameters. The radius $R$ value was computed from 10 images separated by $0.5 \mathrm{~ms}$ and this, for each considered scenario (disk diameter and applied flow rate). This fairly short timescale was chosen in order to show the evolution of sheet holes. To compare them, all the radii $R$ were taken with the same angular coordinates in the lab reference. The radius $R$ was calculated as the difference between the sheet expansion and the hole length. Error bars indicate the standard deviation calculated using 10 measurement repetitions of $R$ based each on 10 images for the same flow rate.

For each disk diameter, the liquid sheet radius increases almost linearly with the $W e$. For the same $Q$, the radius $R$ is higher for a disk diameter decreasing from $40 \mathrm{~mm}$ to $10 \mathrm{~mm}$. This result highlights the effect of the surface friction on the flow. The radius $R$ is increasing linearly with $Q$ despite the appearance of the holes on the sheet corroborated by high lengths error bars. As mentioned above, no benchmarks were found due to a lack of data within the turbulent regime and the evolution trend of the non dimensional critical diameter $\frac{2 R}{d}$ addressed in the introduction is no longer valid.

\subsection{Downstream film velocity}

Fig. 6 presents the mean velocities $U$ of the liquid film measured $1 \mathrm{~mm}$ downstream before the disk edge as a function of $R e$ for three disk diameters. Experimental velocities were obtained knowing the flow rate $Q$ and the measured thickness $h$ of the liquid film. Error bars show the error on $U$ due to the incertitude 


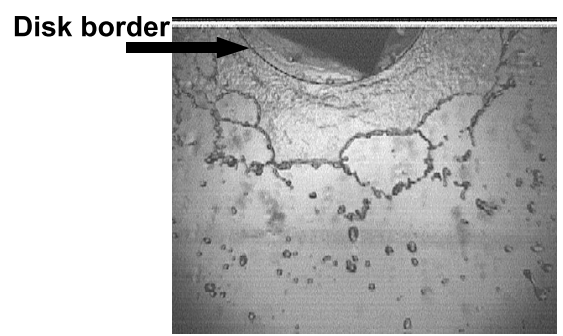

(a)

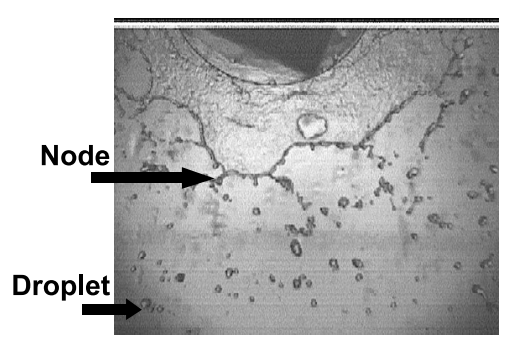

(c)

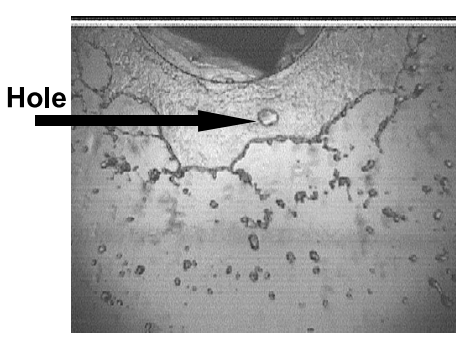

(b)

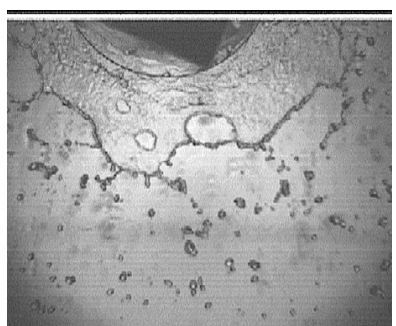

(d)

Fig. 4. Top views of the liquid sheet formed by the perpendicular impact of a water jet on a smooth disk $(D=30 \mathrm{~mm})$. The four successive images (a-d) were timely separated by $0.5 \mathrm{~ms}$. The gap $H$ between the injector tip and the disk surface was $0.55 \mathrm{~mm}$. Perforations appeared next to the plate border, were amplified and then split the sheet leading to droplet formation. The jet was characterized by $U_{0}=4.22 \mathrm{~m} \cdot \mathrm{s}^{-1}, R e=12414$ and $W e=719$.

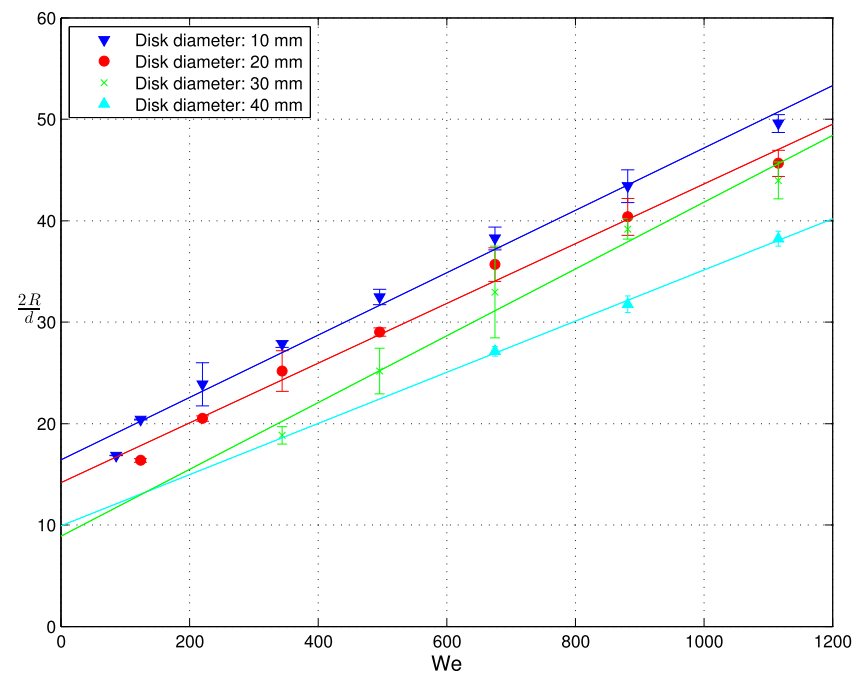

Fig. 5. The behavior of the non dimensional critical diameter $\frac{2 R}{d}$ of tap water sheet as a function of the We and disk diameters. $H$ was set at $0.55 \mathrm{~mm}$. The lines are fit by a linear trend.

on $h$ and the standard deviations of $h$ was established using three independent measurements for the same $R e$. The mean velocity $U$ increases when the $R e$ is increased for each disk diameter. The flow is slowed down when the disk diameter is increased. This can be explained by the increase of friction forces on the disk surface for a given $R e$ what promotes the growth of the boundary layer on the impactor. The liquid surface position was estimated despite the presence of the disturbance waves found at high $R e$. Therefore, the thickness $h$ may have been overestimated since the droplet appears on the needle for the maximum height and not the mean value. This overestimation leads to an underestimation of $U$. When the disk diameter increased to $40 \mathrm{~mm}$, the flow slowed down and disturbances waves was attenuated compared to the impact point. The flow surface was more stable and the measurement process was easier to obtain.

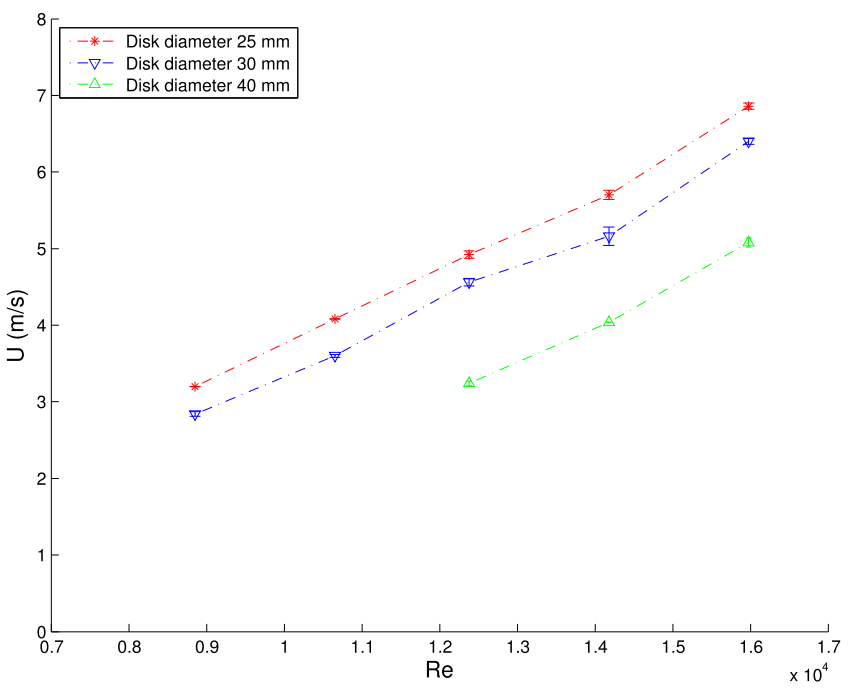

Fig. 6. Velocity $U$ of the liquid flow measured downstream ( $1 \mathrm{~mm}$ before the disk border) as a function of $\operatorname{Re}(\operatorname{Re}$ depends only on the injected $Q$ ).

\section{Impact on a structured disk}

\subsection{Qualitative view}

On the remainder of this study, the disk diameter $D$ was fixed to $30 \mathrm{~mm}$. This diameter $D$ corresponds to the best compromise that allows to have enough space to engrave textures and to keep a sufficient flow rate at the exit of the disk (a larger disk would increase the solid friction experienced by the liquid flow). Rectangular grooves were regularly engraved at the edge of the top disk surface (see Fig. 7) and they had a square section of $1 \mathrm{~mm}$ side and a length $L$ (that was fixed to $5 \mathrm{~mm}$ or $2.5 \mathrm{~mm}$ ). Such grooves can be considered as open channel and are not completely filled by the liquid. Since the grooves were regularly spaced all over the disk perimeter, the disk geometry can be summarized by the number of grooves $N$ and the length of the groove $L$. These parameters allow us to define the gap between two successive grooves as the inside 


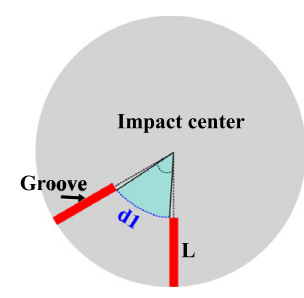

Fig. 7. Representation of the inside circular arc $d 1$ (distance) between two successive grooves. This length is given by $d 1=\left(\frac{D}{2}-L\right) \frac{2 \pi}{N}$ where $D, L$ and $N$ represent respectively the disk diameter $(\mathrm{m})$, the groove length $(\mathrm{m})$ and the groove number.

circular arc between two successive grooves $d 1: d 1=\left(\frac{D}{2}-L\right) \frac{2 \pi}{N}$. Varying $d 1$ and $Q$, four regimes of jet emissions can be observed and they are classified by the number of emitted jets $n$ (drawn in Fig. 8). The number of jets $n$ can be stable or fluctuating.

In the case where the number of jet is stable, two regimes are observed. The first one represents the case where all the liquid is entrapped in the grooves and one can observe one jet per groove, so the number of jets is equal to $N$. The second regime is characterized by the emission of main jets from grooves but also by the presence of secondary jets emitted between two successive grooves. The generated jet number $n$ is then equal to $2 N$. One can notice that, at the disk exit, the main jets seem more turbulent (noisy) compared to secondary jets. This can be explained by the fact that when the liquid film enters in the groove it follows the shape of the groove section. This brutal change in boundary conditions is characterized by the disappearance of the solid influence (friction and wetting property) and by the fact that the shape of the jet is only fixed by the balance between inertia and the capillary forces. At the opposite, the secondary jets are ejected more easily from the disk, since the liquid film just pass between the grooves gap and then converges to form a jet.

In the case for which the number of jets is fluctuating, two regimes are also observed. The first regime represents the case where the secondary jets may locally merge with the main jets. Hence, a mixed zone is formed $\left(n=N_{1}^{*}\right)$. The number of jets $n$ is between $N$ and $2 N$. Finally, even main jets may coalesce and an other mixed zone is formed $\left(n=N_{2}^{*}\right)$. The number of jets $n$ is lower than $N$ and greater or equal to $\frac{N}{2}$. The jet coalescence is elastic in these two cases what explains the fluctuation of the number of jets [26].

A specific symbol for each regime is defined in Fig. 8 and is used in the following paragraphs and especially in the phase diagrams.

\subsection{Phase diagrams}

For a better understanding of the transition between the observed regimes, phase diagrams are determined by varying $Q$ and $d 1$ and are described in the following lines. The first tested configuration is based on grooves with $L=5 \mathrm{~mm}$ (Fig. 9). The different regimes are presented as a function of $d 1$ and $Q$. Each value of $d 1$ appearing on the diagram reflects an independent disk. Below a threshold value in $d 1(=0.12 \mathrm{~mm})$, only main jets are generated and the $n=N$ regime is thus obtained. The distance between the grooves is so small that the liquid is forced to pass through the grooves. In case of high $Q$, the main jets may merge and the number of jets $n$ fluctuates between $\frac{N}{2}$ and $N$ ( $n=N_{2}^{*}$ regime). One states, below the threshold value in $d 1$ and above a $Q=4.110^{-5} \mathrm{~m}^{3} \mathrm{~s}^{-1}$, the grooves are filled and the film liquid is no longer broken up on the disk leading to a Savart sheet with cardioids. Therefore, the regime of Taylor is found [23]. For medium $d 1$ values, main and secondary jets are formed and some of them coalesce. This can be established for all $Q$ ranges in the $n=N_{1}^{*}$ regime. For both high $d 1$

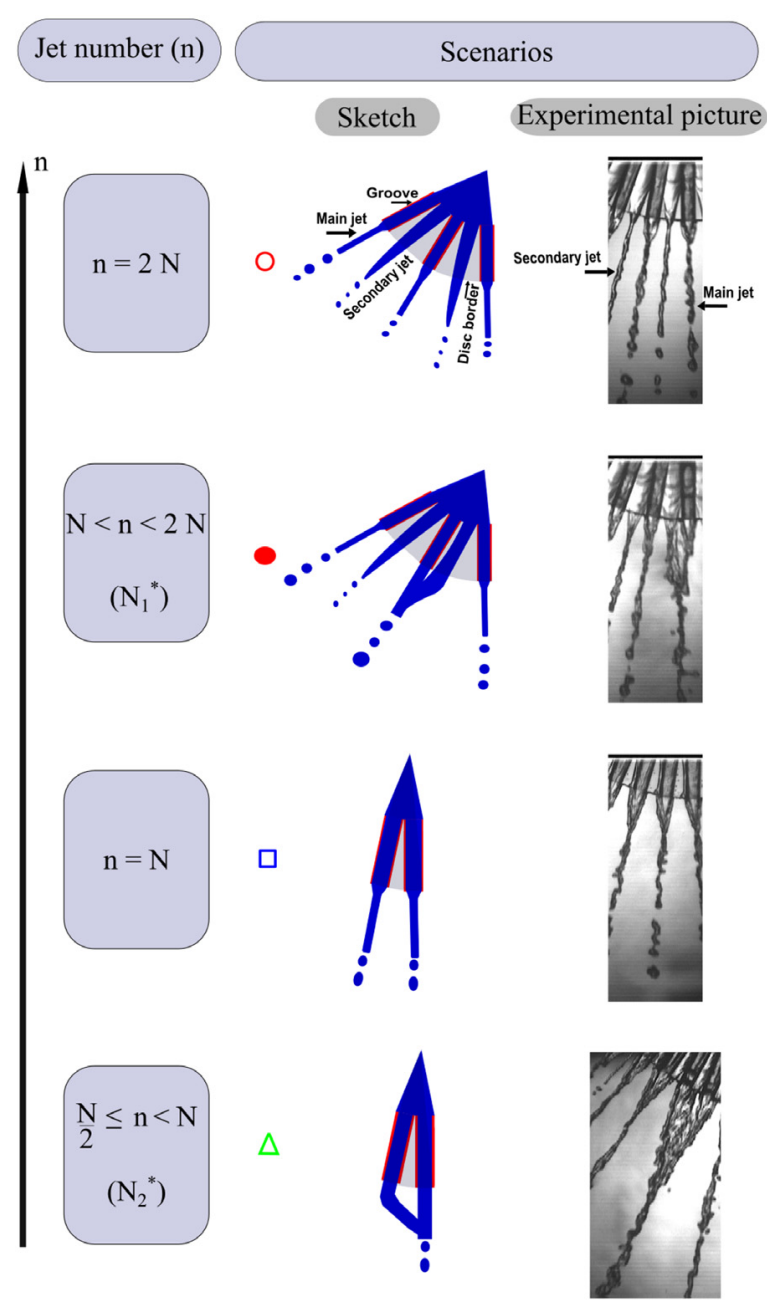

Fig. 8. Different regimes of the liquid behavior on the disk depending on $d 1$ and $Q$. These regimes are classified as a function of the emitted jet number $n$. A specific symbol is given for each regime.

values (>0.42 mm) and low flow rates $Q\left(\leq 210^{-5} \mathrm{~m}^{3} \mathrm{~s}^{-1}\right)$, main and secondary jets are obtained and the jet number $n$ is equal to $2 N$. The secondary jets and main jets may merge with the increase of $Q$ leading to $n=N_{1}^{*}$ regime (mixed zone).

The origin of the secondary jets comes from the destabilization of the liquid sheet by the grooves on the disk. Indeed, for $d 1>$ $0.12 \mathrm{~mm}$ and medium $Q$ values, it is crucial to state that the groove destabilizes early the turbulent liquid film and triggers its break up before reaching the disk edge. The destabilization conducts to the de-wetting of the disk. In Fig. 10(a), a close-up of the disk shows that the secondary jet is surrounded by dry regions along the grooves before being ejected out of the disk. The destabilization of the sheet prior the ejection out of the disk can be also visualized when $d 1$ is large. In Fig. 10(b), between two grooves, one can see a secondary jet. Contrary to the small value of $d 1$ cases, the secondary jets are first liquid sheets as large as the distance between two successive grooves. The turbulent film do not trigger perforations in the formed sheet (Fig. 10(b)). The flow inside this sheet is no longer radial as before the interaction with the grooves but also tangential. These tangential contributions lead to the convergence of the sheet into a jet.

The second proposed configuration is characterized by a groove length reduced by a factor one half from the previous configuration, i.e. $L=2.5 \mathrm{~mm}$. It allows to increase the number of engraved grooves for a disk of the same diameter. As one can see on Fig. 11, 


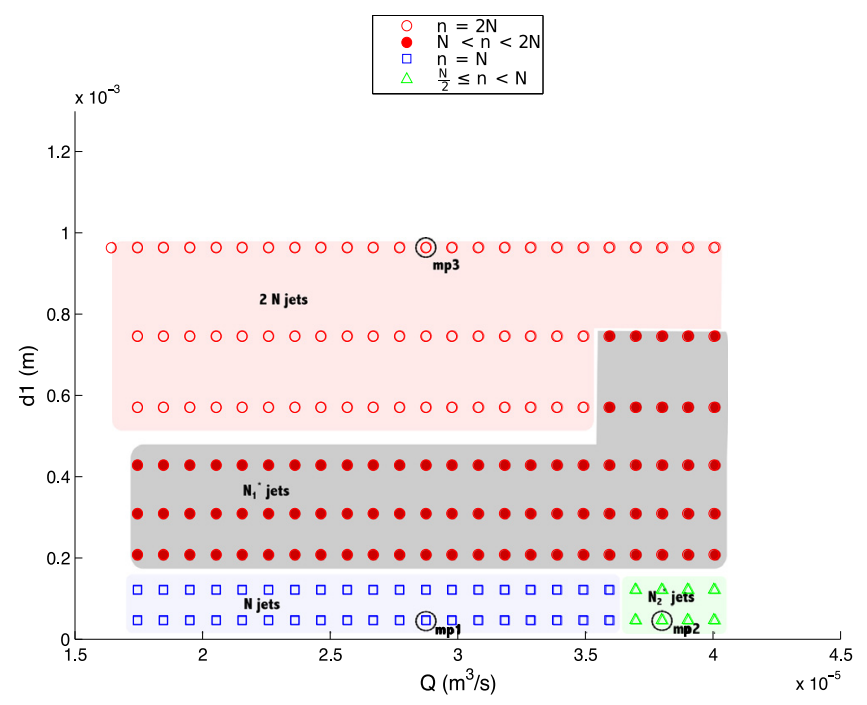

Fig. 9. Representation of different regimes as a function of $d 1(\mathrm{~m})$ and $Q\left(\mathrm{~m}^{3} \mathrm{~s}^{-1}\right)$. The groove length was $5 \mathrm{~mm}$ and the disk diameter $D$ was $30 \mathrm{~mm}$. Three measurement points of the droplet properties (see paragraph 5) were performed and were designed as $m p 1, m p 2$ and $m p 3$.

the transition between the main jet zone ( $n=N$ jets) and the merged zone ( $n=N_{1}^{*}$ ) occurs for the same $d 1$ threshold value as seen in the configuration 1 . This fact confirms that the $n=N$ jet regime is only achieved when the grooves upstream corners are close enough. Then, the transition to $n=N_{2}^{*}$ jet zone is observed for a $Q$ lower than the one observed in the previous configuration. On the contrary, the reduction of the groove length causes an expansion of the mixed zone $\left(n=N_{1}^{*}\right)$ in terms of $d 1$ values and the regime occurs from $0.2 \mathrm{~mm}$ to $0.8 \mathrm{~mm}$. It means that the transitions between the $n=N_{1}^{*}$ and the $n=2 N$ regimes are mainly fixed by the groove length $L$ since the vertical transition happens almost for the same flow rate but with a factor 2 in $d 1$ for the horizontal transition. It is worth noticing that this factor 2 corresponds to the inverse variation in $L$ between the two tested configurations. Furthermore, the $n=2 N$ zone is reduced and is observed for $d 1$ values superior to $0.8 \mathrm{~mm}$. For high $Q$, the main and secondary jets are destabilized and some of them coalesce.

According to the presented results, the generation of individual jets guided by grooves is linked to the groove geometry as well as the gap between them. Below a threshold in $d 1$, one can observe that the incident radial flow is forced to follow the grooves and therefore be guided through these grooves regardless their length $L$. An other important result is that the reduction of the groove length allows to increase the number of emitted jets. However, this length reduction conducts to a lower main jet stability and leads to elastic coalescence phenomena between main and secondary jets. On the other hand, in the confluence region, the resulting jet is excited at a scale defined by the sizes of the incident jets what may increase the noise generated by surface roughness manifested by corrugations. These corrugations constitute the elastic connections areas between two very close jets and hence it affects the number of jets [26].

\section{Characterization of emitted droplets}

One of the goal of the present study is also to address the question of the droplets characteristics, i.e. diameters and velocities. As expected these characteristics are related to the jet emission regime. Here we only focus on the configuration $L=5 \mathrm{~mm}$. To study these characteristics we need to define relevant tools as the mean droplet diameter $D_{m}=\frac{1}{N_{d}} \sum_{i=1}^{N_{d}} D_{d, i}\left(N_{d}\right.$ : the droplet
Table 1

Characteristics of the measurement points of different zones.

\begin{tabular}{lll}
\hline & $d 1(\mathrm{~mm})$ & $Q\left(10^{-5} \mathrm{~m}^{3} \mathrm{~s}^{-1}\right)$ \\
\hline$r e f$ & - & 2.8 \\
$m p 1$ & 0.047 & 2.8 \\
$m p 2$ & 0.047 & 3.8 \\
$m p 3$ & 0.963 & 2.8 \\
\hline
\end{tabular}

number, $D_{d}$ : the droplet diameter), the Standard deviation STD = $\sqrt{\frac{\sum_{i=1}^{N_{d}}\left(D_{d, i}-D_{m}\right)^{2}}{N_{d}}}$ and the coefficient of variation $C V=\frac{S T D}{D_{m}}$.

The Fig. 12 presents the probability density function (PDF) of droplets where droplet percentages are plotted as a function of droplet diameters for four different situations: The reference measurement ref corresponds to droplets emitted from the liquid sheet (Disk without grooves and $D=30 \mathrm{~mm}$ ) and the measurement points $m p 1, m p 2$ and $m p 3$ correspond respectively to $n=N$ jet zone, $n=N_{2}^{*}$ jet zone and $n=2 N$ jet zone (Fig. 9). Characteristics of the measurement points are shown in Table 1.

In the case of ref, the droplet size distribution has a probability peak corresponding to droplet diameters of $300 \mu \mathrm{m}$ with and $D_{m}=572 \mu \mathrm{m}$. The distribution is wide and droplet diameters range between $150 \mu \mathrm{m}$ and $2800 \mu \mathrm{m}$; this result is consistent with the measurements of Clanet and Villermaux [9]. The extent of the distribution is illustrated by high STD and $C V$ values (respectively $374.33 \mu \mathrm{m}$ and $65 \%$ ). One founds low percentages (inferior to $2 \%$ ) of probabilities to obtain droplets with a diameter between 650 and $2800 \mu \mathrm{m}$. For all the situations involving an engraved disk, i.e. $m p 1, m p 2$ and $m p 3$, the droplet diameter ranges are narrower than the ref case. In these regimes, the shape of the droplet size distribution is characterized by two probability peaks reflecting a bimodal aspect. One can thus consider for each measurement points a main (largest peak) and a secondary (lowest peak) droplets populations. For all the measurement points ( $m p 1, m p 2$ and $m p 3$ ), the secondary droplets population peak appears to be close to the main peak of the ref curve but less pronounced than the ref case. Droplet diameters exceeding $1000 \mu \mathrm{m}$ have very low percentages and correspond to the merged droplets which is not always the case for the ref measurement point.

For the measurement point $m p 1$ (corresponding to $n=N$ jet regime), the main droplet population is centered on a droplet diameter value of $585 \mu \mathrm{m}$ and the secondary droplet population is characterized by smaller droplets with diameters close to $250 \mu \mathrm{m}$. The reduced amplitude for the droplet diameter distribution can be quantified by low STD and $C V$ values which are respectively 183.19 $\mu \mathrm{m}$ and $31 \%$.

Furthermore, for $m p 2$ which corresponds to an increase of $Q$ and a transition to $n=N_{2}^{*}$ jet zone, the PDF curve keeps the same shape with two distinct droplet populations. In contrast with $m p 1$, one can observe that secondary probability peak (small droplet diameter) increases and reaches a peak of $9.47 \%$. This value is then almost the same than the one corresponding to the primary peak and this has for effect to reduce the mean droplet diameter $D_{m}$ to $503 \mu \mathrm{m}$. The unexpected increase of small droplets proportion may be explained by collisions between main jets which conduct, as a result, to more droplets created by an other phenomenon than the Plateau-Rayleigh destabilization. This droplet size distribution is highlighted by higher STD and CV values (respectively $196.71 \mu \mathrm{m}$ and $39 \%$ ). The decrease of $D_{m}$ with the increase of $Q$ corresponds to that of Clanet and Villermaux [9].

For the measurement point $m p 3$ (corresponding to $n=2 \mathrm{~N}$ jet regime), the mean droplet diameter value $D_{m}$ is $554 \mu \mathrm{m}$ and the percentage of small droplets slightly decreases compared to $m p 1$. One can also remark that the main probability peak is higher and that the droplet distribution is tightened around this peak. Both phenomena conduct to low STD and $C V$ values which are 


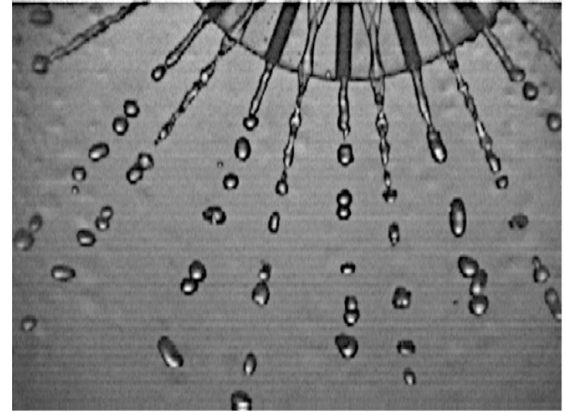

(a)

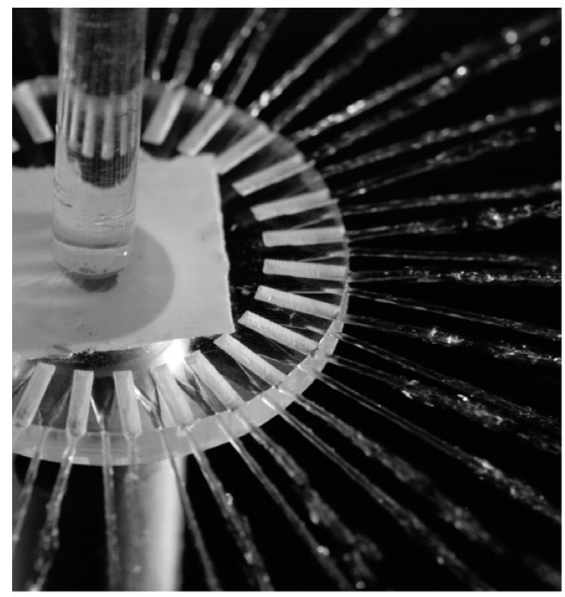

(c)

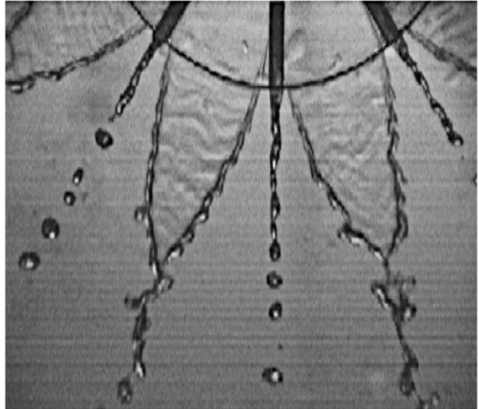

(b)

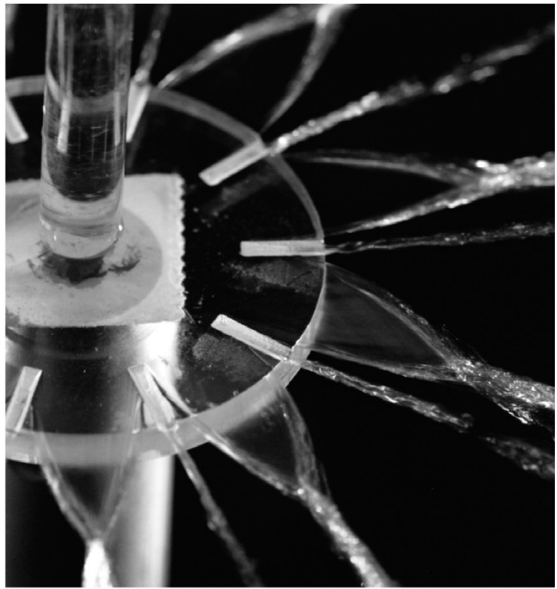

(d)

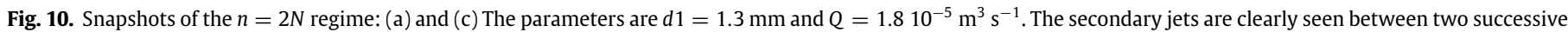

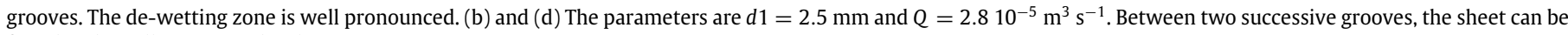
found and it collapses into droplets.

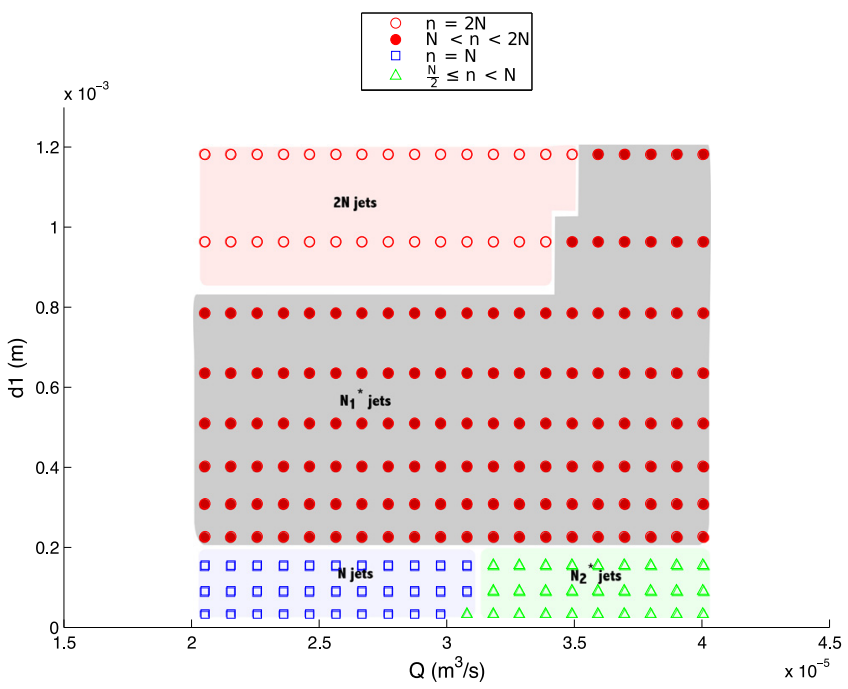

Fig. 11. Representation of different regimes as a function of $d 1(\mathrm{~m})$ and $Q\left(\mathrm{~m}^{3} \mathrm{~s}^{-1}\right)$. The groove length was $2.5 \mathrm{~mm}$ and the disk diameter $D$ was $30 \mathrm{~mm}$.

respectively $155.62 \mu \mathrm{m}$ and $28 \%$. This result may be explained by the fact that the secondary jet emitted between the groove gap is less noisy than the liquid guided by the groove. According to $\mathrm{mp} 1$ and $m p 3$, it is possible to generate similar droplet size distributions with close $D_{m}$ regardless of the groove geometry.

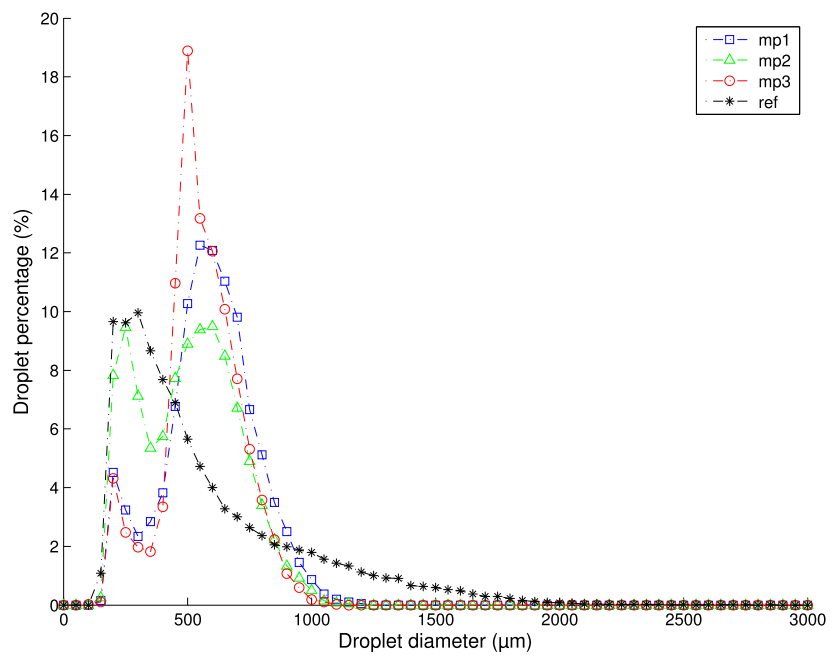

Fig. 12. Representation of droplet percentages as a function of their diameters for three measurement points $m p 1, m p 2$ and $m p 3$. The curve ref is the reference measurement which corresponds to droplets emitted from the liquid sheet (without grooves).

Following the Plateau-Rayleigh instability, the droplet diameter is 1.89 times the jet diameter and thus one can expects the mean droplet diameter $D_{m}$ to be close of $1.89 \mathrm{~mm}$ for droplets emitted from a jet issued from a $1 \mathrm{~mm}$ side square section groove. The discrepancy between this expected $D_{m}$ and the ones measured can 


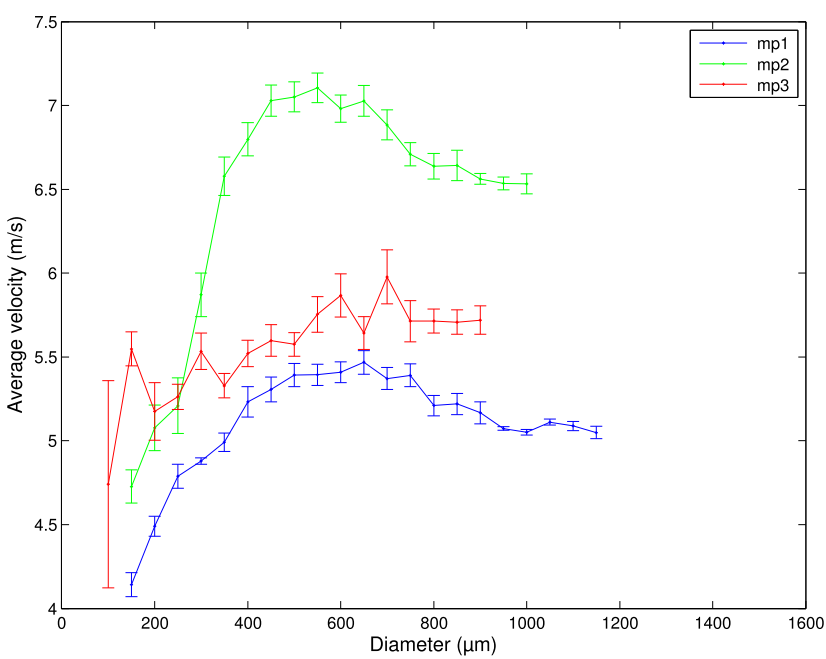

Fig. 13. Droplet velocities as a function of their diameters for three measurement points $m p 1, m p 2$ and $m p 3$.

be explained by the fact that the grooves are not totally filled by the liquid and thus the liquid ejected from the grooves converges in jets smaller than the output grooves sections.

The phenomenon of merging jets was investigated by Eggers and Villermaux [26]. Although, the case addressed by these authors presented different technical aspects as they explored two smooth jets within an inelastic merging with a high angle of collision between the two jets, some similarities were clearly observed. In both studies, the droplet size distribution of the resulting jet was extended compared to a single jet. It is noticeable that our droplet size distribution of the resulting jet presented two populations of droplets, which was less visible in their PDF curves. This behavior was strongly related to the noise resulting during the turbulent jets collision. Following this trend, the stronger the noise, the broader the droplet size distribution [26].

Velocities of emitted droplets for $m p 1, m p 2$ and $m p 3$ are measured and are presented as a function of their diameters (Fig. 13). Each velocity per diameter class corresponds to the average velocity of 15 droplets. For the measurement point $m p 1$ ( $n=N$ jet regime), the velocity curve is divided in two parts. The first part, for small droplet diameters (up to $400 \mu \mathrm{m}$ ) is characterized by an abrupt slope, velocities varying between $4.2 \mathrm{~m} \mathrm{~s}^{-1}$ to $5 \mathrm{~m} \mathrm{~s}^{-1}$ for larger diameters. The second part of the curve is characterized by an almost constant velocity. The first increasing part can be understood as the diminution of the air friction contribution to the drop motion when the droplet diameter increases. For $m p 2$ ( $n=N_{2}^{*}$ jet regime), the curve presents a similar shape and droplet velocities increase as expected since the increase of $Q$. A slight velocity decrease corresponding to droplet ranges exceeding 700 $\mu \mathrm{m}$ is however observed. This could be explained by the merging phenomena that slow down the resulting droplet. Moreover, for $m p 3$ ( $n=2 N$ jet regime), the velocity curve is similar to the one obtained in the $n=N$ jet regime (mp1) with the same $Q$. Except that velocities are slightly increased. It appears that the regime change (and particularly the apparition of secondary jet) has no real effect on the droplets velocities. It is also noticeable that droplet velocities correspond almost to film velocity measured above. For example, in the case of $D=30 \mathrm{~mm}$, the mean droplet velocities corresponding to $m p 1$ and $m p 3$ tested at $Q=2.810^{-5}$ $\mathrm{m}^{3} \mathrm{~s}^{-1}(R e=12407)$ are respectively $5 \mathrm{~m} \mathrm{~s}^{-1}$ and $5.5 \mathrm{~m} \mathrm{~s}^{-1}$ and are close to that one measured in the disk border $\left(4.5 \mathrm{~m} \mathrm{~s}^{-1}\right)$. The difference is linked to the measurement technique of the mean velocity.

\section{Conclusion}

This study investigates a turbulent round water jet impacting a motionless disk. A turbulent thin film is formed and flows radially. The flow velocity $U$ downstream ( $1 \mathrm{~mm}$ before the disk edge) rises linearly with the Re for the different disk diameters. The flow is slowed down when the disk diameter is increased due to the increase of solid friction for a given Re. When the flow exceeds the disk edge, a Savart liquid sheet is obtained and its radius increases almost linearly with $Q$ despite some holes affecting the sheet.

Furthermore, the influence of engraved grooves on the disk border is investigated regarding the droplet production. We have proved that the groove was able to control the turbulent sheet by splitting the film in multiple jets before reaching the disk edge. Phase diagrams presented as a function of the parameters $d 1$ and $Q$ were useful to highlight the transition between jet regimes $(n=$ $2 N, n=N_{1}^{*}, n=N$ and $n=N_{2}^{*}$ ). It is noticeable that the $n=N$ jet regime is directly linked to the disk geometry and particularly to the space between grooves. One states also, even for high $d 1$ and $Q$, the turbulent flow do not trigger local disturbances downstream the groove and a stable sheet between two neighboring grooves (with no perforations) is generated outside the disk that leads to jet (Fig. 10(b)). Moreover, the reduction of the groove length leads to generate a large number of jets. However, in this situation, the main and secondary jets are less stable what may increase the merging phenomenon. We have also characterized the distribution in diameters for the emitted droplets. It appears that the mean diameter $D_{m}$ of these droplet size distributions is still large (around $500 \mu \mathrm{m}$ ) with respect to the industrial interest. Finally, we found that droplet velocities corresponds almost to the film velocity $1 \mathrm{~mm}$ before the disk border.

To sum up, this study deals with an industrial application that concerns the dispersion of pesticide. The main objective is to narrow the droplet size distribution compared to conventional atomizer used in agriculture. This goal is reached by splitting the liquid film formed on the solid target using engraved radial grooves. In such a way, several smaller liquid jets are formed instead of a circular liquid sheet leading to narrower drop size distributions.

\section{Acknowledgments}

We thank the University of Liege-Gembloux Agro-Bio Tech and in particular TERRA - AgricultureIsLife's platform for funding this research project. S.D thanks the support from FNRS as Senior Research Associate.

\section{References}

[1] G. Matthews, Pesticide Application Methods, John Wiley \& Sons, 2008.

[2] S. Ouled Taleb Salah, N. De Cock, M. Massinon, B. Schiffers, S. Dorbolo, F. Lebeau, Étude des potentialités des systèmes d'application contrôlée des gouttes (CDA) pour les traitements phytosanitaires en céréaliculture (synthèse bibliographique), in: Biotechnologie, Agronomie, Société et Environnement= Biotechnology, Agronomy, Society and Environment, 2016.

[3] R. Rioboo, M. Voué, A. Vaillant, J. De Coninck, Drop impact on porous superhydrophobic polymer surfaces, Langmuir 24 (24) (2008) 14074-14077.

[4] M. Massinon, F. Lebeau, Experimental method for the assessment of agricultural spray retention based on high-speed imaging of drop impact on a synthetic superhydrophobic surface, Biosyst. Eng. 112 (1) (2012) 56-64.

[5] H. Boukhalfa, M. Massinon, M. Belhamra, F. Lebeau, Contribution of spray droplet pinning fragmentation to canopy retention, Crop Prot. 56 (2014) 9197.

[6] F. Savart, Mémoire sur la constitution des veines liquides lancées par des orifices circulaires en mince paroi, Ann. Chim. Phys. 53 (337) (1833) 1833.

[7] F. Savart, Mémoire sur le choc d'une veine liquide lancée contre un plan circulaire, Ann. chim. 54 (56) (1833) 1833.

[8] C. Clanet, Les nappes d'eau de félix savart, Bull. National SFP 125 (2000) 11-15.

[9] C. Clanet, E. Villermaux, Life of a smooth liquid sheet, J. Fluid Mech. 462 (2002) $307-340$. 
[10] E. Villermaux, C. Clanet, Life of a flapping liquid sheet, J. Fluid Mech. 462 (2002) $341-363$.

[11] J.M. Gordillo, H. Lhuissier, E. Villermaux, On the cusps bordering liquid sheets, J. Fluid Mech. 754 (2014) R1.

[12] J.A.F. Plateau, Statique expérimentale et théorique des liquides soumis aux seules forces moléculaires, vol. 2, Gauthier-Villars, 1873.

[13] L. Rayleigh, On the capillary phenomena of jets, Proc. R. Soc. Lond. 29 (196199) (1879) 71-97.

[14] C. Dumouchel, On the experimental investigation on primary atomization of liquid streams, Exp. Fluids 45 (3) (2008) 371-422.

[15] C. Clanet, Dynamics and stability of water bells, J. Fluid Mech. 430 (2001) 111147.

[16] G. Taylor, The dynamics of thin sheets of fluid. III. Disintegration of fluid sheets, Proc. R. Soc. Lond. Ser. A Math. Phys. Eng. Sci. 253 (1274) (1959) 313-321.

[17] E. Watson, The radial spread of a liquid jet over a horizontal plane, J. Fluid Mech. 20 (03) (1964) 481-499.

[18] T. Azuma, T. Hoshino, The radial flow of a thin liquid film: 2nd report, liquid film thickness, Bull. JSME 27 (234) (1984) 2747-2754.
[19] N. De Cock, M. Massinon, S.O.T. Salah, B. Mercatoris, M.R. Vetrano, F. Lebeau, Dynamics of a thin radial liquid flow, Fire Saf. J. 83 (2016) 1-6.

[20] T. Bohr, P. Dimon, V. Putkaradze, Shallow-water approach to the circular hydraulic jump, J. Fluid Mech. 254 (1993) 635-648.

[21] A. Duchesne, L. Lebon, L. Limat, Constant Froude number in a circular hydraulic jump and its implication on the jump radius selection, Europhys. Lett. 107 (5) (2014) 54002

[22] E. Dressaire, L. Courbin, A. Delancy, M. Roper, H.A. Stone, Study of polygonal water bells: Inertia-dominated thin-film flows over microtextured surfaces, J. Fluid Mech. 721 (2013) 46-57.

[23] Taylor, The dynamics of thin-sheets of fluid. I. Water bells, Proc. R. Soc. A 253 (1274) (1959) 289-295.

[24] N. De Cock, M. Massinon, D. Nuyttens, D. Dekeyser, F. Lebeau, Measurement of reference ISO nozzles by high-speed imaging, Crop Prot. 89 (2016) 105-115.

[25] G. Thunivumani, H. Gadgil, Dynamics of liquid sheet breakup in splash plate atomization, J. Fluids Eng. 140 (1) (2018) 011205

[26] J. Eggers, E. Villermaux, Physics of liquid jets, Rep. Progr. Phys. 71 (3) (2008) 036601. 